\title{
Archéopages
}

Archéopages

Archéologie et société

43 | 2016

Médecines

\section{Le sanctuaire antique du site des Jacobins au Mans}

Des eaux pour guérir et pour maudire

The ancient sanctuary of the Jacobins site in Le Mans. Waters to heal and curse El santuario antiguo del sitio de los Jacobinos en Le Mans. Aguas para curar y maldecir

Pierre Chevet

\section{CpenEdition}

Journals

Édition électronique

URL : https://journals.openedition.org/archeopages/1691

DOI : 10.4000/archeopages. 1691

ISSN : 2269-9872

Éditeur

INRAP - Institut national de recherches archéologiques préventives

Édition imprimée

Date de publication : 1 novembre 2016

Pagination : 18-23

ISSN : $1622-8545$

\section{Référence électronique}

Pierre Chevet, «Le sanctuaire antique du site des Jacobins au Mans », Archéopages [En ligne], 43|

2016, mis en ligne le 01 décembre 2018, consulté le 02 juin 2021. URL : http://

journals.openedition.org/archeopages/1691; DOI : https://doi.org/10.4000/archeopages.1691 


\section{Le sanctuaire antique du site des Jacobins au Mans \\ Des eaux pour guérir et pour maudire}

Pierre Chevet Inrap
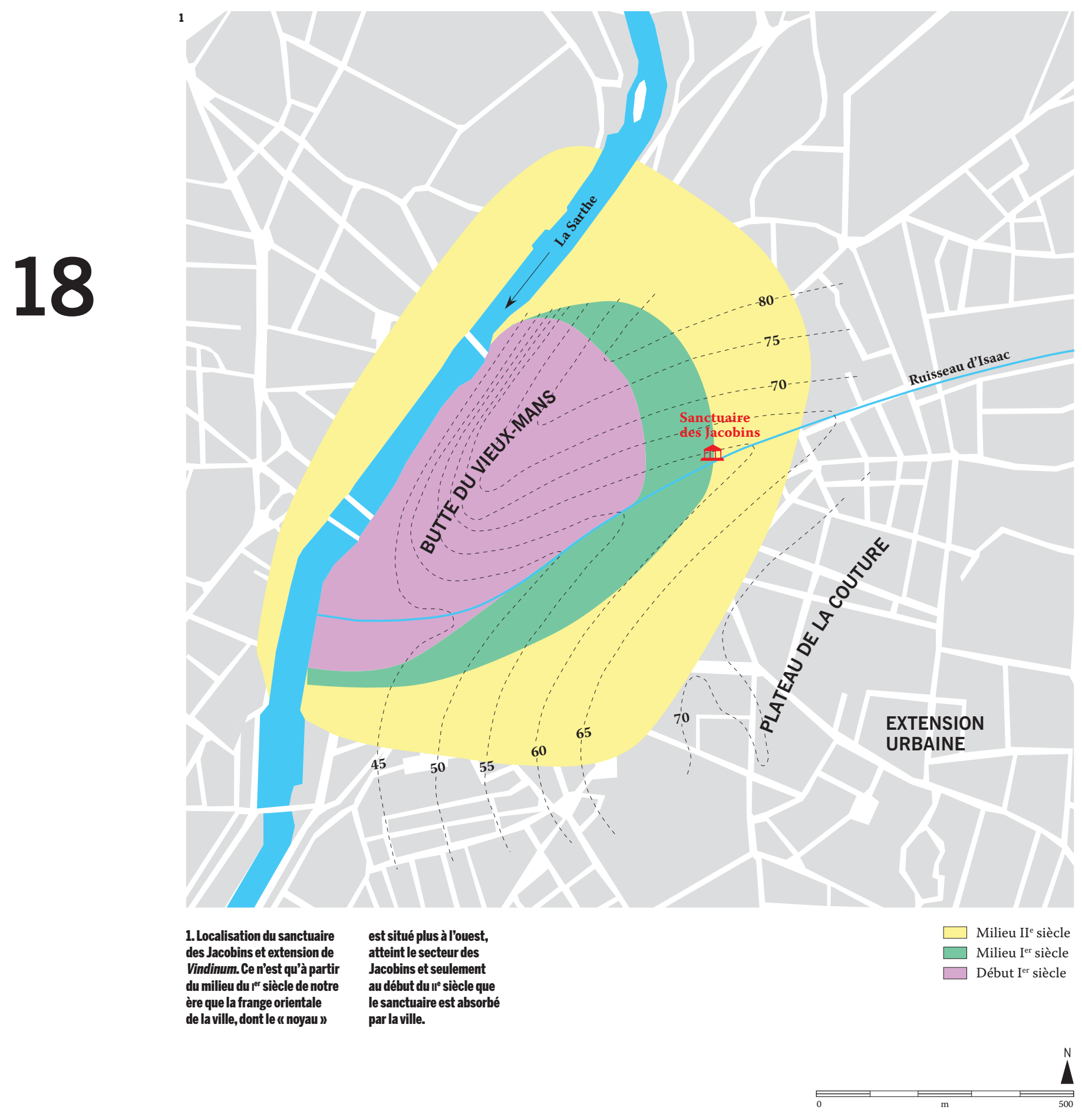
La fouille préventive préalable à la construction du nouvel espace culturel des Jacobins au Mans ${ }^{1}$ a conduit à la mise au jour, en plein centre ville, d'un quartier urbain de Vindimum (nom de la ville antique du Mans, alors capitale de cité des Aulerques cénomans). Outre trois rues le long desquelles se répartissent habitations et bâtiments fonctionnels, cette intervention a surtout été l'occasion de révéler l'existence d'un sanctuaire des eaux (Chevet et al., 2015-2016), déployé de part et d'autre du lit de l'ancien ruisseau d'Isaac qui, au début de notre ère, traversait le site de part en part.

Si la découverte de lieux dédiés à une divinité aquatique n'est pas, en soit, particulièrement exceptionnelle, ce qui fait l'originalité du sanctuaire du Mans est son insertion dans le tissu urbain de Vindinum. De tels espaces sacrés sont en effet presque systématiquement établis dans un cadre naturel, souvent remarquable par tel ou tel aspect, d'où la croyance qu'une divinité s'y est installée. Létude du site des Jacobins montre en fait que le cas du Mans n'échappe pas totalement à cette règle puisque l'origine du lieu sacré, qui remonte à la fin de lâge du Fer, nous place à une époque où aucun peuplement aggloméré ne se développe à proximité [ill. 1].

\section{Un culte remontant à la conquête romaine}

L'identification du lieu sacré repose en particulier sur la récolte de nombreuses monnaies, dont certaines de forte valeur, tant dans le lit du ruisseau lui-même que sur ses berges. L'hypothèse d'un gué, lieu où les offrandes monétaires sont fréquentes, doit être exclue et c'est sans doute la conjonction entre une topographie en replat et le comportement irrégulier et brusque du ruisseau, dont le débit peut plus que décupler en cas d'épisode pluvieux sévère, qu'il faut mettre en avant pour justifier la dévolution du lieu à une divinité aquatique. Il faut en effet imaginer un site brutalement inondable et parcouru par des eaux tumultueuses, à la suite par exemple d'un violent orage, tous éléments en mesure de lui conférer le caractère remarquable évoqué supra ce qui, dans la mentalité antique, est interprété comme le signe d'une présence divine.

Le segment chronologique couvert par les quelque 600 monnaies d'offrande récoltées permet de fixer assez précisément la date des premiers dons, qui débutent autour de 50 avant notre ère, voire peut-être un peu avant, mais il convient de rester prudent à ce sujet. À cette époque, le site est très peu aménagé et la rive gauche du ruisseau est totalement déserte. En rive droite, seuls une petite occupation ponctuelle et un four de potier sont établis au nord-ouest du site. Le secteur nord-est, correspondant à la zone sacrée, se caractérise par la présence d'un numéraire gaulois ou républicain abondant, témoignant d'offrandes réalisées de part et d'autre et dans le lit du ruisseau [ill. 2A] qui reste alors, et pour encore plusieurs décennies, dans un environnement naturel exempt de toute transformation. En l'état des connaissances, rien ne permet d'affirmer une relation fonctionnelle entre le lieu de culte et l'occupation qui le borde à l'ouest, toutes les hypothèses à ce sujet sont recevables.

\section{Un étang sacré et un habillage architectural}

Ce n'est que durant la première moitié du $\mathrm{I}^{\mathrm{er}}$ siècle de notre ère, probablement à partir des années $15 / 20$, que le site cultuel connaît ses premières modifications, avec notamment la fixation de la section aval du lit du ruisseau dans un chenal artificiel et la construction, en rive gauche, d'un petit édicule votif, sorte d'autel/tronc ou de chapelle dans laquelle se concentrent les offrandes monétaires jusqu'alors cantonnées au niveau de ruisseau [ill. 2B et 3]. Dès lors, et parallèlement au déploiement de Vindinum qui atteint petit à petit le site sacré, l'ampleur des modifications va s'accroître. La plus importante consiste en la création d'un plan d'eau artificiel qui occupe tout le quart nord-est de la fouille, soit plus de $2000 \mathrm{~m}^{2}$ [ill. $2 \mathrm{C}$ ]. Les centaines de monnaies et objets à fonction votive récoltés dans les dépôts de vase de cette petite retenue lui confèrent une vocation cultuelle particulièrement affirmée et justifient son appellation d'étang sacré (Chevet et al., 2014). Il matérialise la résidence de la divinité aquatique censée habiter le lieu et se maintiendra jusqu'à la fin de l'histoire du sanctuaire, qui connaît une dernière transformation d'importance avec la création, sans doute vers 140, d'un mur péribole délimitant une aire sacrée incluant l'étang, le petit édicule et sans aucun doute un temple à part entière, situé hors de l'emprise de la fouille [ill. 2D]. Ce dernier habillage architectural contribue à totalement isoler l'espace consacré tout en l'intégrant dans le tissu urbain de la ville, qui à cette époque a totalement cerné le site.

À partir de la fin du II ${ }^{\mathrm{e}}$ siècle, on assiste à un phénomène d'envasement généralisé du fond de vallée, ce qui contribue à l'ennoiement du site cultuel et à son abandon. Une fois l'étang totalement colmaté, puis asséché artificiellement durant le $\mathrm{III}^{\mathrm{e}}$ siècle par une dérivation au sud du ruisseau d'Isaac [ill. 2E], le lieu conserve malgré tout sa valeur sacrée : aux jets de monnaies et d'objets dans le plan d'eau ou l'autel/tronc se substituent de nouveaux rituels, avec notamment l'enfouissement de céramiques contenant des offrandes alimentaires (Chevet et al., 2014 et 2015-2016; Raux et al., 2015).

\section{Un sanctuaire guérisseur}

Le sanctuaire des Jacobins compte parmi ceux qui ont livré des ex-voto anatomiques, témoignant vraisemblablement des vertus guérisseuses accordées aux eaux de l'Isaac et l'inscrivant ainsi au rang des trois ou quatre sanctuaires guérisseurs cénomans parmi les vingt-deux sites cultuels actuellement identifiés dans cette cité ${ }^{2}$ (Raux, 2015).

Malheureusement, aucune information ne permet pour l'heure d'identifier directement la divinité qui habitait les eaux du site. Cependant, 

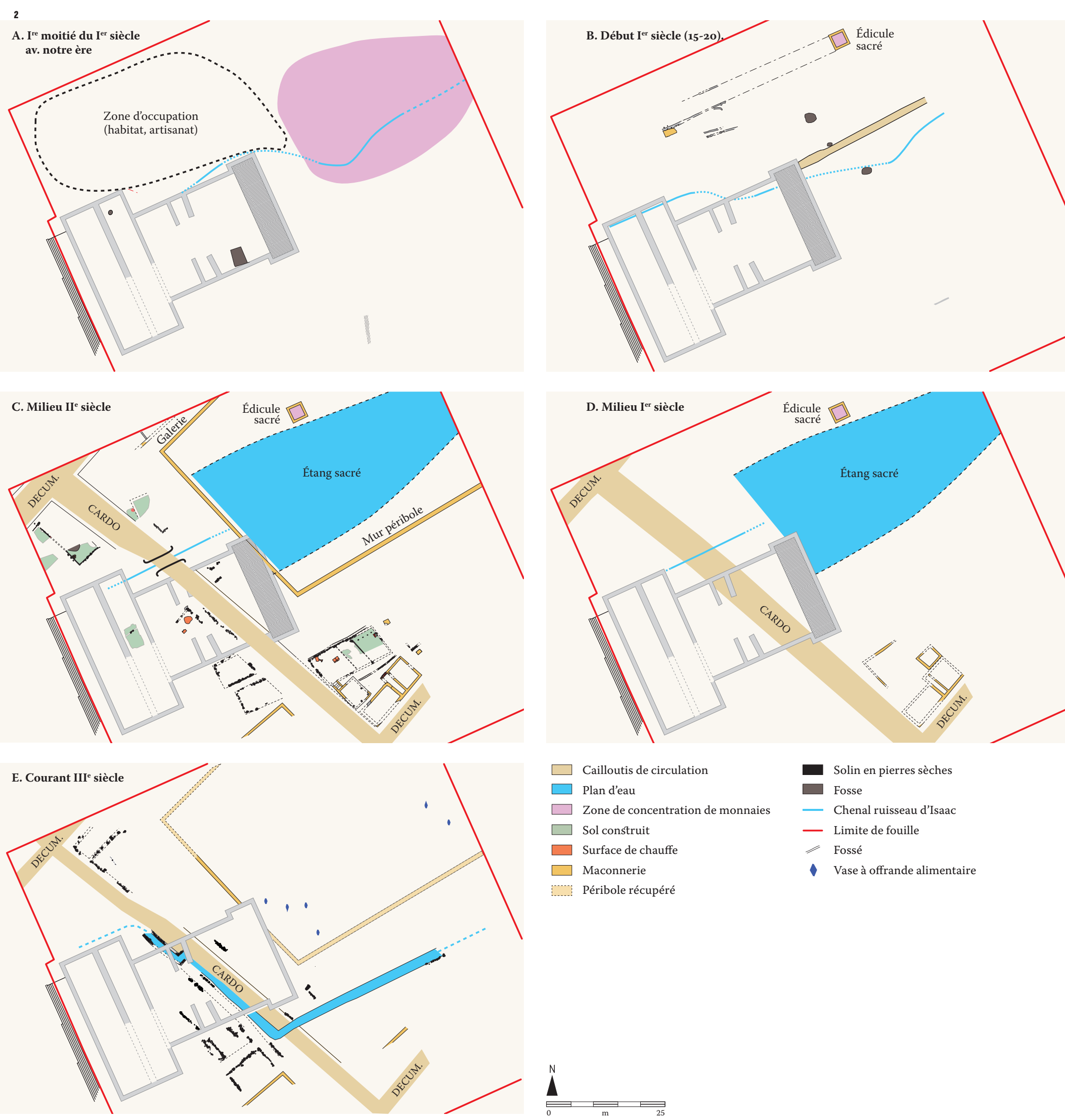

Solin en pierres sèches

Fosse

_ Chenal ruisseau d'Isaac

_ Limite de fouille

Fossé

- Vase à offrande alimentaire 
la question liée à la dimension curative des eaux du sanctuaire des Jacobins trouve peut-être un élément de résolution avec la découverte, à $400 \mathrm{~m}$ du sanctuaire, de deux bases de statues mentionnant le dieu Apollon, connu en Gaule pour sa fonction de medicus (qui chasse les maladies), et sa parèdre indigène, Sirona [ill. 4]. Ces blocs épigraphiés ayant été remployés dans la base de l'enceinte du Bas-Empire, il est bien sûr délicat d'assurer leur lien avec le sanctuaire des Jacobins. Pour autant, l'hypothèse est chronologiquement recevable, l'édification de cette enceinte succédant de peu au démantèlement du sanctuaire.

\section{Des monnaies pour les dieux...}

On l'aura compris, tout au long de la fréquentation du sanctuaire, le jet de monnaies (la iactatio stipis) reste le geste rituel dominant. L'objet de cette pratique, ainsi que, d'une façon plus générale, de toute offrande, est de remercier la divinité d'un bénéfice perçu ou de la solliciter pour en obtenir une faveur. Si la quantité et la concentration des monnaies récoltées ne suffisaient à se convaincre de ce caractère rituel, on peut s'appuyer sur la composition du corpus monétaire pour emporter la conviction. Le lot présente en effet un certain nombre de caractéristiques qui démontrent sans équivoque sa nature votive, avec par exemple une surreprésentation des petites divisions monétaires, ou encore la présence de monnaies de substitution en plomb, de rondelles... S'il est certes important d'honorer la divinité du lieu, beaucoup de fidèles le font selon leurs moyens et souvent à moindre frais ce qui, a contrario, n'empêche pas les plus riches d'entre eux d'offrir parfois des monnaies d'argent de plus forte valeur [ill. 5], elles aussi en surnombre par rapport à la masse monétaire en circulation usuelle. Cet ensemble monétaire est caractéristique des contextes d'offrandes, le geste symbolique prenant probablement le pas sur la valeur réelle de l'offrande, ainsi qu'en témoignent les monnaies de substitution, dont l'usage monétaire est évidemment totalement exclu.

Au côté des monnaies, on trouve également toute une palette d'objets habituels en contexte religieux, qu'il s'agisse d'objets de dévotion ou d'instruments propres au fonctionnement du monument. Pour le premier groupe, il s'agit notamment d'instruments de toilette, d'objets de parure telles des bagues, fibules [ill. 6] ou chaînettes parfois en or [ill. 7], ou encore d'objets de valeur en métal précieux [ill. 8], qui rendent compte d'offrandes de natures diverses, mais toujours réalisées dans le but d'honorer ou de remercier la divinité. Parmi les autres découvertes à dimension non sacrée reflétant la vie quotidienne du monument, on citera plusieurs clochettes (tintinnabula [ill. 9]), et des boîtes à sceau et stylets d'écriture (sur tablettes de cire), qui correspondent à des objets usagés ou perdus, en lien avec l'activité comptable et administrative des desservants du sanctuaire, lesquels comptaient à l'époque parmi les rares lettrés.

\section{Et du plomb pour les maléfices}

Côtoyant ces offrandes et objets usuels, reflets de la religion officielle qui se pratique au grand jour, le sanctuaire des Jacobins se distingue par une pratique beaucoup plus occulte et réprouvée par Rome, se référant à la magie noire en ce sens qu'elle reste secrète et cachée. La fouille a ainsi permis la découverte d'une douzaine de tablettes de défixion (de defixio = envoûtement). Il s'agit de feuilles de plomb sur lesquelles sont gravées des formules d'envoûtement. La feuille est repliée sur elle-même et jetée dans l'étang sacré, charge ensuite à la divinité d'exaucer le sort. Sur le site du Mans, la plupart de ces tablettes sont illisibles car inscrites dans un langage inventé (symbolique), incompréhensible pour les hommes, mais censé être parfaitement clair pour les dieux. Une seule tablette «bien écrite » porte un long texte actuellement en cours de traduction [ill. 10], espérons qu'elle nous apprendra le nom de la divinité qui occupait les eaux de l'Isaac.

Il n'est pas sans ironie de constater qu'alors que la religion offre des dieux et des édifices précis pour porter soins et guérison à ses fidèles, les lieux sacrés sont également utilisés secrètement pour sceller des maléfices parmi lesquels malheurs et maladies ne peuvent que figurer!

.


3. Ce petit édifice votif carré

de $3 \times 3 \mathrm{~m}$ a été construit au

début du $\mathrm{I}^{\mathrm{er}}$ siècle de notre ère

à proximité de l'étang sacré.

4. En l'état des connaissances,

ces bases de statues

d'Apollon et Sirona (H. 0,61 et

$0,54 \mathrm{~m}$ ) restent la meilleure

piste pour approcher

la question du patronage

des eaux du sanctuaire.

5. Deniers d'argent des

$I^{\text {er }}$ siècle avant notre ère

et $\mathrm{I}^{\text {er }}$ siècle de notre ère

(de gauche à droite, Titius

quintus, Auguste et Galba).

Avec les gros bronzes de fort

valeur également retrouvés

lors de la fouille, ces

monnaies permettent de

démontrer la qualité sacrée des eaux de l'étang.

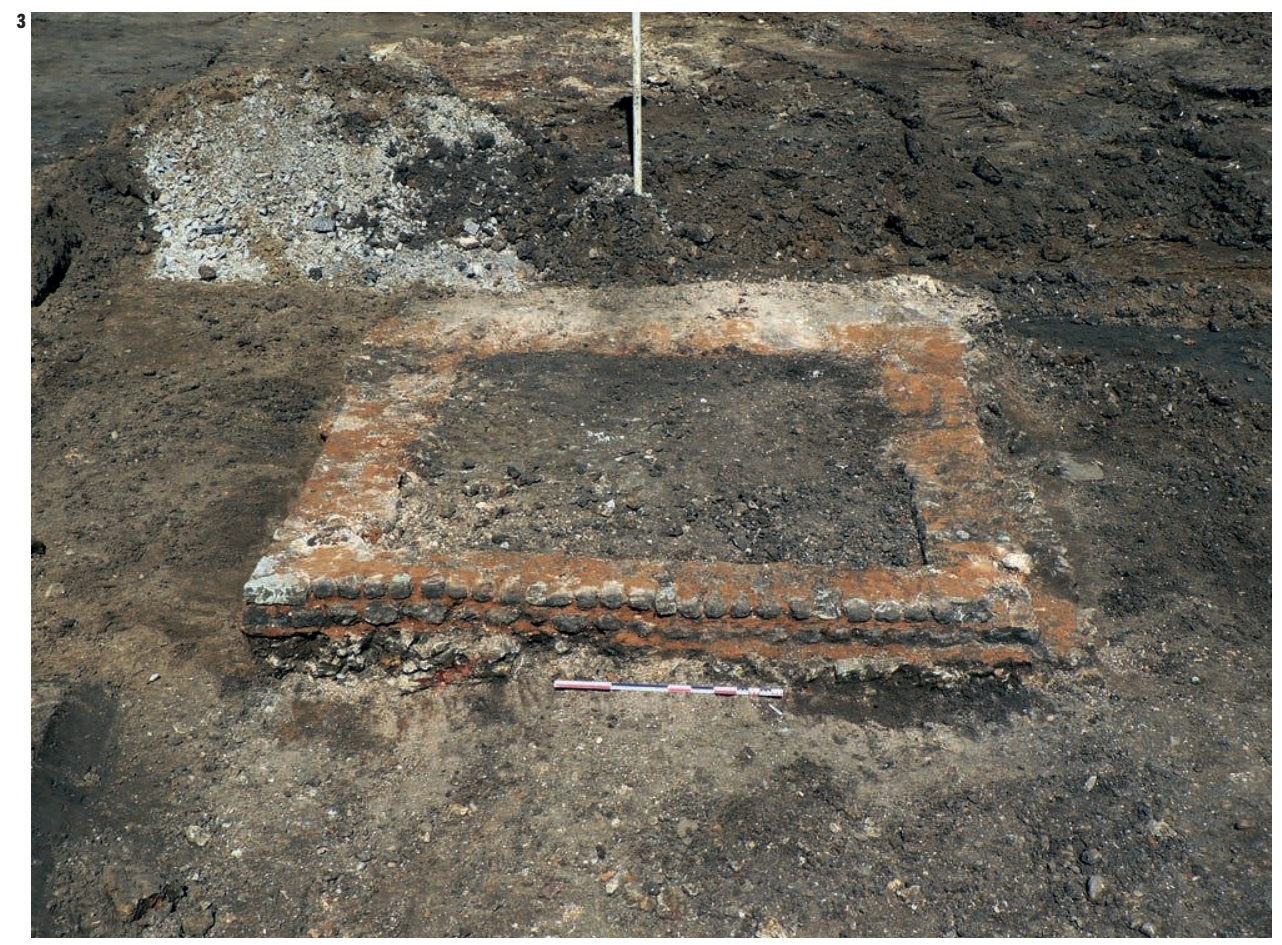

$\approx$
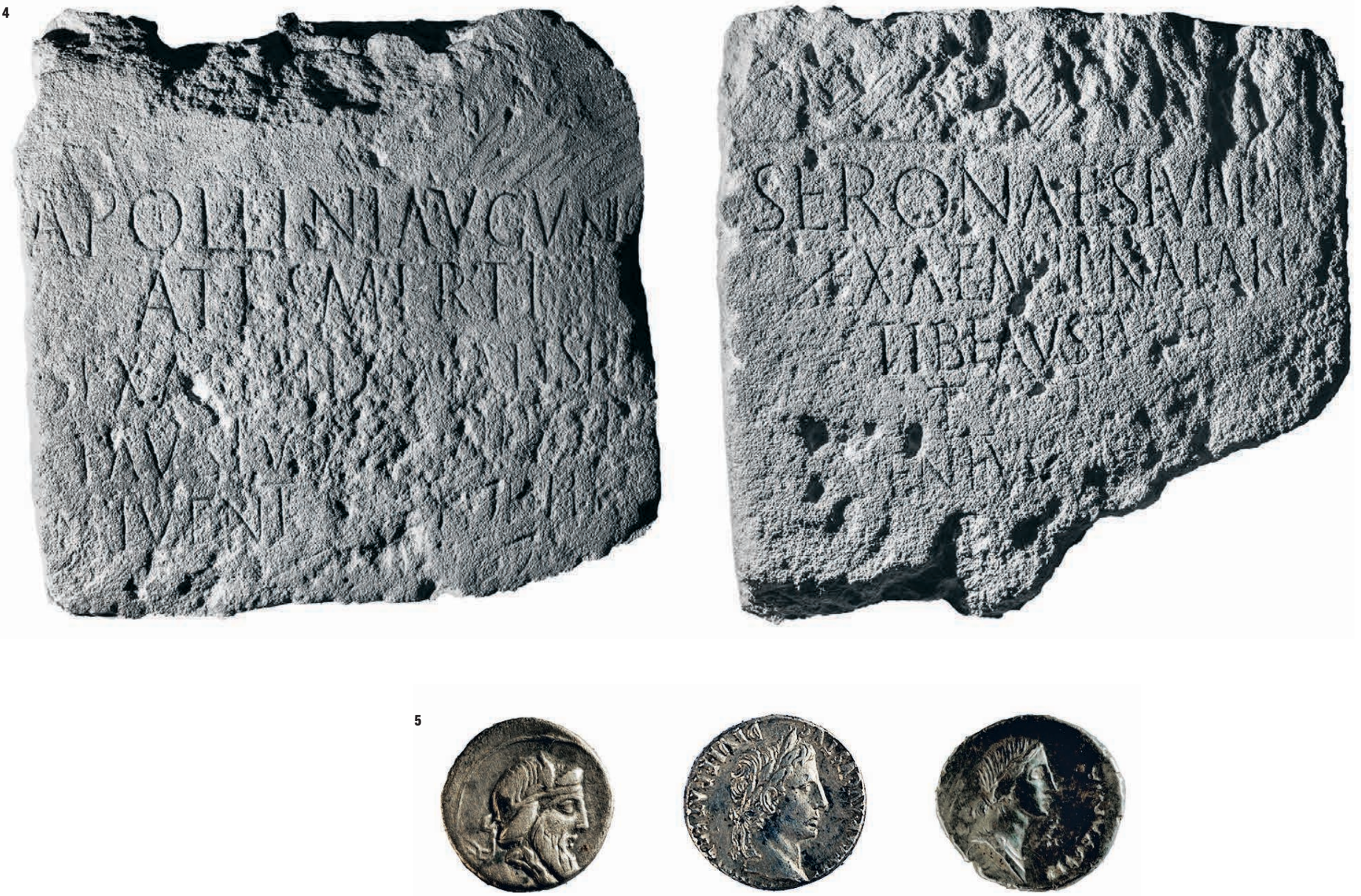
6. Fibules à charnières en alliages cuivreux (de gauche à droite et bas en haut:

L. 45 mm, 37 mm, 55 mm

et $29 \mathrm{~mm}$ ). De gauche à droite

et haut en bas : fibule dite

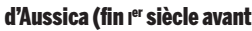

notre ère-début ${ }^{\text {er }}$ siècle de

notre ère), fibule émaillée sur

arc rectangulaire (II siècle),

fibule d'Aussica type dérivé

(milieu I Ir siècle-II siècle

de notre ère), fibule émaillée

sur arc zoomorphe figurant

un lièvre ou un lapin (II' siècle

de notre ère).

7. Bague et éléments de

chaînette en or (Diam. 15 mm,

17/2/17 mm). La présence

de ces éléments en métal

précieux témoigne d'une

sollicitation appuyée auprès

de la divinité, que l'on

récompense en conséquence.

8. Cuillère en argent

(L. $142 \mathrm{~mm}$ ). Objet également

précieux, ce bel objet

en argent porte la même

signification que les

anneaux en or.

(H. 47,23 et $26 \mathrm{~mm}$ )

Comme cela se pratique

encore dans les sanctuaires

polythéistes d'Extrême-

Orient, les clochettes

servent notamment à attirer

l'attention de la divinité

Iorsque le fidèle s'adresse

à elle.

10. Tablette de défixion

(L. totale : $80 \mathrm{~mm}$ ) en plomb,

métal associé aux puissances

chtoniennes et maléfiques,

destinée à porter malheur

à un rival ou un ennemi.
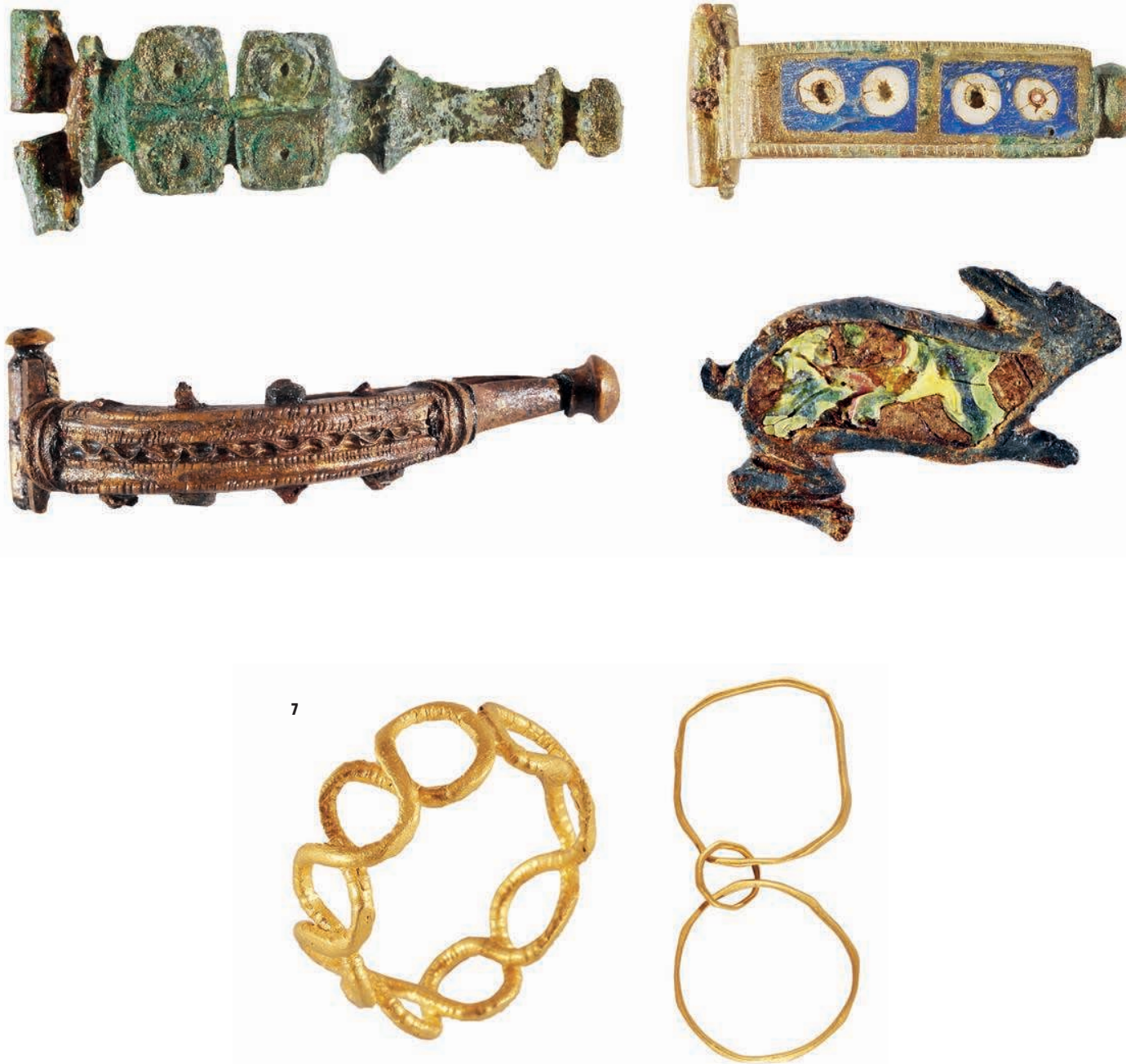

กุ

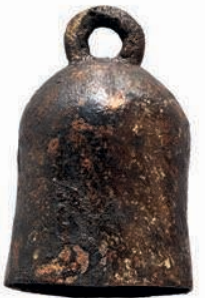

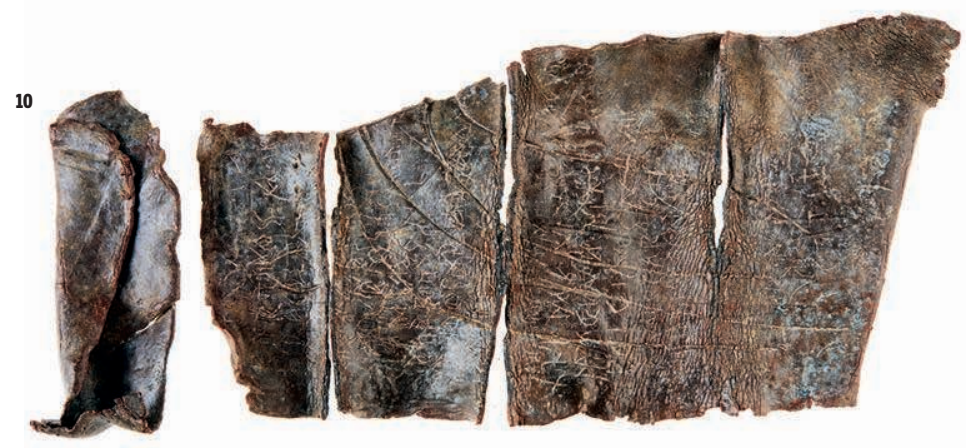
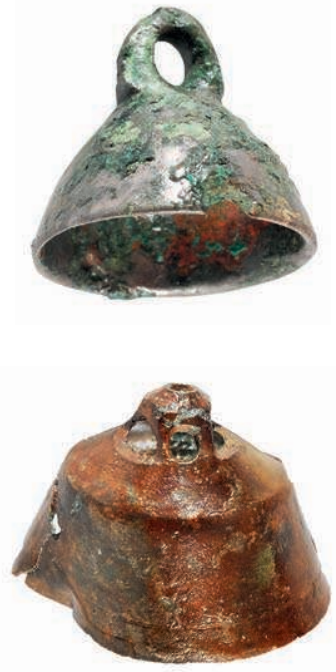